\title{
Perceiving Stress on Affective State Based on Features from EEG
}

\author{
Hafizuddin Muhd Adnan, Hamwira Yaacob, \\ Kulliyyah of Information \& Communication Technology, \\ International Islamic University Malaysia, Kuala Lumpur, Malaysia, \\ hafizuddin.adnan@live.iium.edu.my \\ Kulliyyah of Information \& Communication Technology, \\ International Islamic University Malaysia, Kuala Lumpur, Malaysia, \\ hyaacob@iium.edu.my
}

\begin{abstract}
The EEG (Electroencephalogram) from brain signal has been broadly used to reveal human affects based on Valence, Arousal and dominance through computational modelling. Recently, less study on EEG been done in detail, directly to reveal and quantify the stress from affects dynamically based on EEG signal. In addition. There is no study currently perform to identify stress from the features based on EEG signals simultaneously. As for the objective of this paper, this study will only try to do features analysis of stress by comparing the statistical features of affects from the data obtained from three subjects. The data was collected during the trial session for new assembled EEG mobile system at Simulation Centre Akademi Laut Malaysia (ALAM). Subjects consist of three marine pilot who were given tasks to navigate ships in the simulated scenes. Findings and observations from the analysis are reported in this paper.
\end{abstract}

Keywords- EEG; Stress; Affective State.

\section{INTRODUCTION}

Stress terminology were defined into many aspects and terms, for instance in term of Biology, Mechanical Engineering and Psychology area [20]. Stress commonly correlated to human affects in understanding [13], [27], therefore the precise definition for stress in this study is according to Psychology aspects. In Psychology, stress was defined as any uncomfortable emotional experience which is attended by probable biochemical, physiological and behavioural changes [5] while a person experiences in response to threat and an external stimulus. Stress become very serious health crisis nowadays [4] which lead to affected to public work performance. Currently, most of study in stress were done in psychology area [7], [9], very less study on stress were done in neurophysiological in computational studies using EEG signal [11], [15].

EEG (electroencephalogram) is defined as a graphical representation of the brain's electrical activity and one of the neuroimaging tools. EEG systems record electrical potentials from various locations on the scalp using some specific devices. These potentials are generated by neural activity within the brain. Common categorizations of frequencies in EEG include alpha $(8-13 \mathrm{~Hz})$, beta $(13-30 \mathrm{~Hz})$, gamma $(30-600 \mathrm{~Hz})$, delta $(<4 \mathrm{~Hz})$, theta $(4-8 \mathrm{~Hz})$, and $\mathrm{mu}$ $(8-13 \mathrm{~Hz})$ [1]. Theta and alpha frequency bands are generally used for studying affects (emotions) [16], [17] From the signal of EEG been captured from the device, it goes through on some process lead to the respond to the affective state.

With relevant to the Affective Stimulation in this study, according to [30], to classify happiness, sadness, neutral and fear affective states, they were captured by EEG signals through channels $\mathrm{F}_{3}$ and $\mathrm{F}_{4}$, over theta and alpha bands. EEG signals captured at $\mathrm{C}_{3}, \mathrm{C}_{4}, \mathrm{~F}_{3}, \mathrm{~F}_{4}, \mathrm{P}_{5}, \mathrm{P}_{6}, \mathrm{~T}_{7}$ and $\mathrm{T} 8$ are only captured from alpha frequency band [34].

Currently, in computational studies, some studies on stress were done using EEG to measure the stress level, and the model were developed for the stress detection [19], [32], [34]. Even though, yet the consequence of study shows that there is no study perform to identify stress from the features based on EEG signals. Hence, it is generally not agreed upon which features from EEG most appropriate for affective state recognition [18]. Therefore, it seems crucial for study that can give the immediate outcome for stress detection from EEG signal and simultaneously convey the information for the stress profiling. In addition, from the previous study, 
the data collected from EEG signal to detect and measure the stress level, for example, study done by [32] only using single channel of electrode. By means of this, the result is not feasible to generalize the stress level as the other EEG channels were neglected.

Therefore, the objective of this study is to analyze the statistical features on EEG signal and the to identify by perceiving and point the maximum value, minimum value, the mean and standard deviation value from the statistical features which were mostly correlated to the stress. Then this study will report the pre- analysis on EEG data obtained from the subject by analyzing the statistical features from the EEG data through the resting state and affective state

This paper consists of six sections. The abstract summarized the study. Section one, introduction explain about the definition of stress and EEG. Then the problem statement and objective of study. Section two describe the design of study, explained in detail about the experiment protocol, data and data analysis. Section three discuss on the results and some discussion related to the result observed from the graphs and figures. Then section four is the conclusion, discussed on analysis and proposed on the future study to complement. Section five is the acknowledgment to those support and involved in this study. Then lastly, section sic is the list of references.

\section{STUDY DESIGN}

Specific EEG device system which is assembled and turn it into the term of mobility was used for data collection (Figure 1), namely EXG Brain Marker. This device is a high-quality device with a CE- certified. This device accomplished to measure up to 19 channels for EEG signal. The signal derived from this device in term of (signal-to-noise ratio) is extremely high, thus the system highly relevant for scientific research as well as standard applications in hospitals and practices. The weight of this device is under 500 grams. It is operated with power adapter with 12 volts with 2 to 4 amps (Ampere) The current produces by this device were with the medical grade of power supply. It protects the patient from any harm when using it with electrodes. This EXG device use the electrodes cap (Wave guard connect). The electrode cap consists of two universal size which is for kids and adults. The electrodes are connected accordingly to the EXG electrodes port based on the international standard positioning system (10-20 system). The electrode port at the EXG Brain Marker device with the universal size standard (DIN Standard) which allowing enabling the use of most available electrodes [11] This device was assembled for the purpose of dynamic data collection whereas the subjects or participant can move along together while completing the given task.

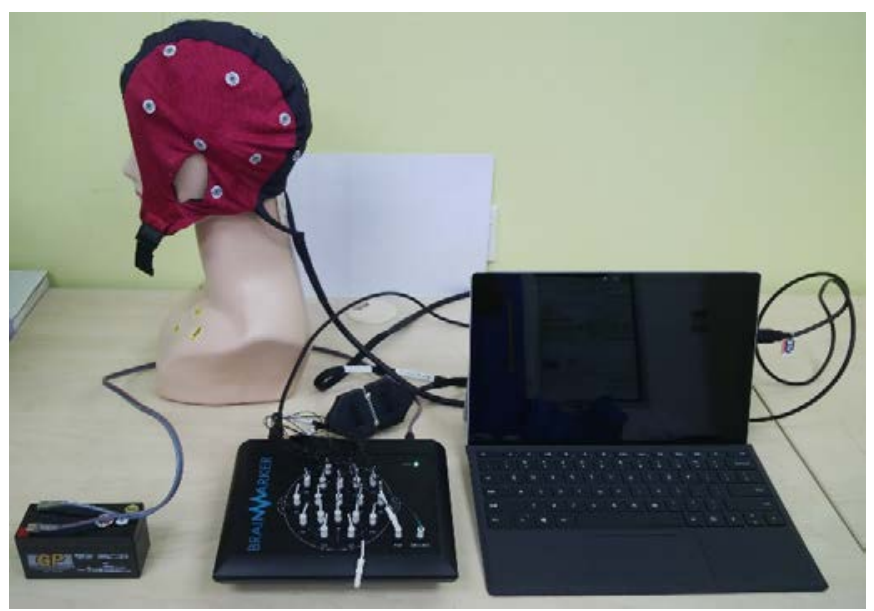

Figure 1. EXG Brain Marker Mobile EEG Device

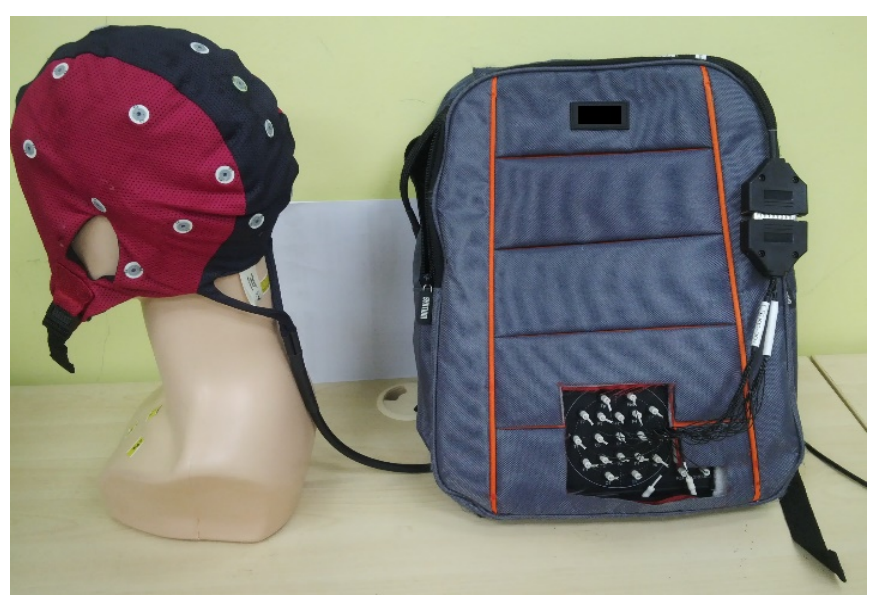

Figure 2. EXG Brain Marker Mobile EEG Device in Bag pack.

In this study, combination and additional device were used. They are, Surface Pro 4 with specification of Intel Core i5 processor, GB RAM,128GB SSD for storage,12.3 Inch display and weight 800 Grams. Then the portable rechargeable battery with 12 volt and 2.28 ampere. This battery can last for six hours with continues usage. Lastly the portable Wi-Fi with $4 \mathrm{~g}$ connection speed, subscribed to local Malaysia telco company.

\section{A. EXPERIMENTAL PROTOCOL}

Data collection was performed at ALAM (Malacca Marine Academy), the subject is cargo ship pilot. The data were recorded during the simulation practice by the pilot. The pilot run the simulation practice based on the given and setup scenario.

The protocol or the paradigm of task sequence for the participant is divided into three main tasks (Figure 3 ). While 
performing all the tasks, the data are recorded using the EXC Brain Marker device. Formerly before performing the tasks, as a protocol for data collection, the participant will fill up the consent form and the answers the questionnaire for personality and stress test.

\section{Paradigm of Task Sequence}

Task 1
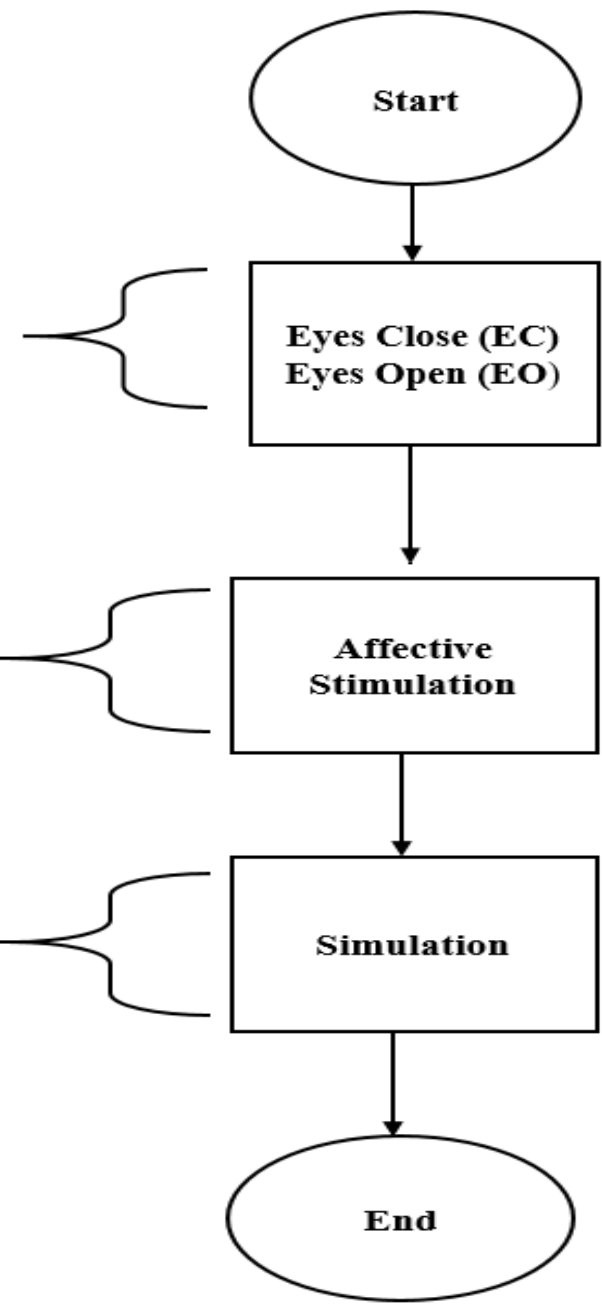

Task 2

Figure 3. The Paradigm of Task Sequence

1) EYES OPEN AND EYES CLOSE (RESTING STATES): The figure 5 shown the task sequence involved. The first is eyes close and eyes open task (Resting States) [35]. The participant was asked to sit and closed their eyes for one minute. Later, open their eye looking at the blank white screen for 1 minute.

2) AFFECTIVE STATE STIMULATION: The second task is the affective stimulation. Participant was asked to watch the affective stimulation slide for four minutes. Participant would watch the IAPS slide for 4 minutes, the content of slide consists of images reflect to calm, happy, fear and sad. IAPS stand for International Affective Picture System (IAPS; Center for the Study of Emotion and Attention, 1994) [22], [23].

In this study, the IAPS were selected from a set of static images based on a dimensional model of emotion. The image set comprises various pictures depicting mutilations, snakes, insects, attack scenes, accidents, contamination, illness, loss, pollution, puppies, babies, and landscape scenes, among others. According to [28], [36], [42] based on some dimensional model of emotion, it is generally shown that models including only two dimensions, valence and arousal, which are superior to models including more than these two dimensions. Consequently, the IAPS in this study select the images that will reproduce happy, calm, fear and sad. The selected images reflect to the valance and arousal in the categories of positive emotion (happy, calm) and negative emotion (sad, fear). Based on valence and arousal, the study can identify the subject for the stress detection level.

3) Pilot Simulation: The third task for experiment is the pilot simulation event at the Simulation Centre. The setup as a real marine ship complete with all control panel device and equipment at the cockpit deck (Figure 9). The scenario for simulation was projected through the surrounded screen glass in front of the cockpit. Participant will run the simulation where the scenario had been set up for the evaluation their action controlling the ship. The data recoding starts immediately when the simulation run. Common duration for a simulation is around 45 minutes. When the simulation stop, data recoding will be stop immediately to tally with the scenario time.

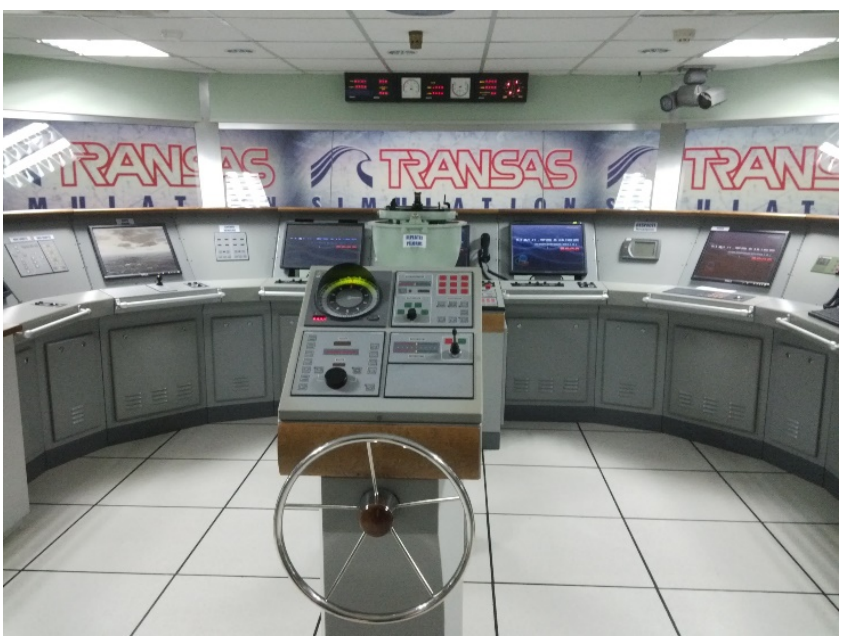

Figure 4. Simulation Layout

\section{B. DATA}

The data were attained and recorded from participants from three marine pilots at ALAM (Akademi Laut Malaysia) Simulation Centre. The pilot consists of novice, competent 
and expert for their ranking. Participant from novice pilot had 1 years' experience and the competent pilot had 5 years' experience and the expert is 27 years' experience in marine pilot.

The subjects never been as subjects in any EEG experiments study and this is their first time participating. Thus, the data collection session setup took more times for the instruction and explanation about each protocol session going through.

\section{ANALYSIS}

In this section, the study will describe how each form of data was pre-processed and then analyzed.

1) DATA PREPARATION: The recorded EEG data from the EXG brain Marker device been classified as raw data. Data will be go through pre-processing method to get the clean data. As for recorded data during the simulation session, the data was processed through training process, which is consist of filtering, feature extraction and classification.

For filtering, the raw data from any noise and artefact to get a clean data base on it respected frequency band which is Delta, Theta, Alpha and Beta. By filtering, it removes most of the artifacts and remove all high frequency noise.

Then it went through feature extraction to extract the emotional state which is consist of happy, Calm, sad and fear. Then the classification process to classify the emotional state according to the Valence and Arousal. The trained data (emotional data) were test with the value of Valence and arousal to get the NET and it accuracy.

Then the resting state data (eyes open and eyes closed) were processed into two-part, filtering and feature extraction (spectrogram). After that the Resting state data been compared with the NET from emotional data to get the result of definite emotional state according to Valence and Arousal. Lastly, the study refers to the participant personality and stress test questionnaire result and keep it as a record for further study on it.

2) STATISTICAL FEATURES: EEG signals are the rich source of information about brain activity including affective state. It need to extract the valuable information from this large amount of data by reducing the amount of data available because normally, it is not possible to use these data for emotion recognition This process is known as feature extraction. This process extracts specified measures that are useful for the task. Features are characteristics of EEG signal that can distinguish between different emotions [14]. The signals were decomposed into the four commonly bands, Delta $(\mathrm{oHz}, 4 \mathrm{~Hz})$, Theta $(4 \mathrm{~Hz}, 7.5 \mathrm{~Hz})$, Alpha $(8 \mathrm{~Hz}$, $13 \mathrm{~Hz})$, Beta $(13 \mathrm{HZ}, 30 \mathrm{~Hz})$. This study focuses on Frequency Domain for features extraction, through the PSD (Power
Spectral Density) method to extract features for four affective state, happy, calm, sad and fear regarding to the Valence and Arousal. The features from the EEG signals been analyzed for each channel from 19 channels. This is to observe the statistical features from EEG signals for the maximum, minimum, mean and standard deviation and then compare it between three participants or subjects. This is to spot which channel is mostly correlated to the affective state that tend to lead to the stress.

\section{RESULT AND DISCUSSION}

The EEG data in this study were processed using MATLAB software. As for statistical features comparison, we use Microsoft Excel to analyse the value for maximum, minimum, mean and standard deviation for 19 channels from 3 participants. In this paper we only show the result from the resting state only from three participants because we already get the result of definite emotional state according to Valence and Arousal.

The following figure will show on maximum, minimum, mean and standard deviation for the resting state (eyes close and eyes open) for three different participants.

\section{A. EYES CLOSED}

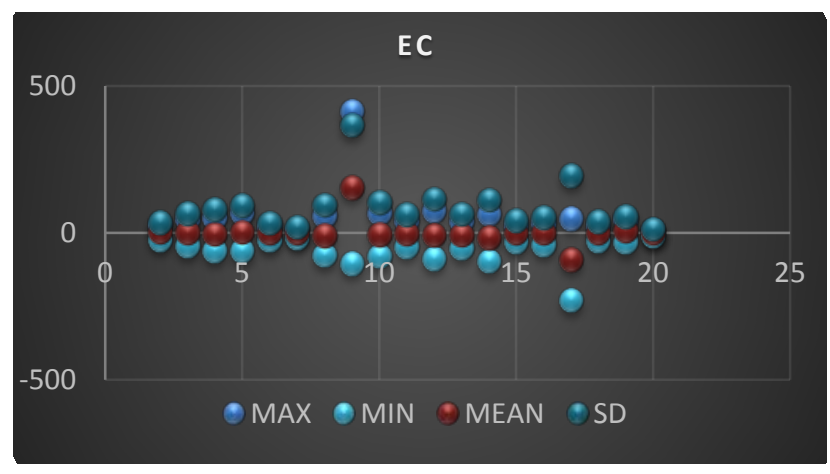

Figure 5. Participant 1

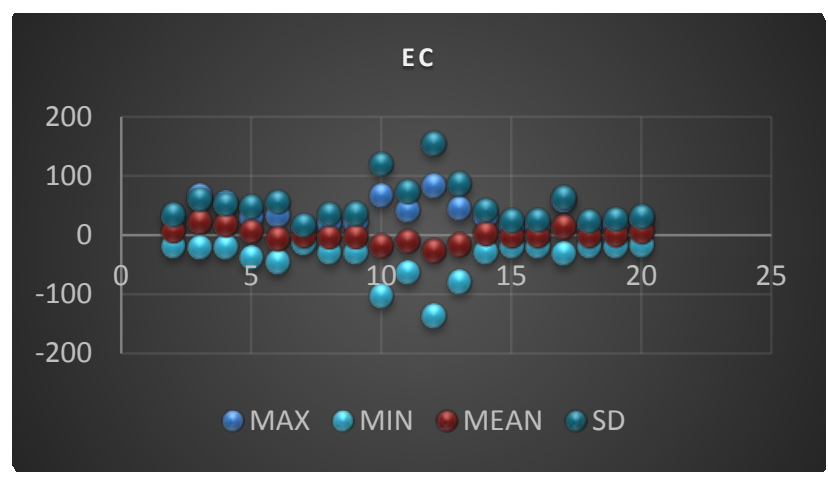

Figure 6. Participant 2 


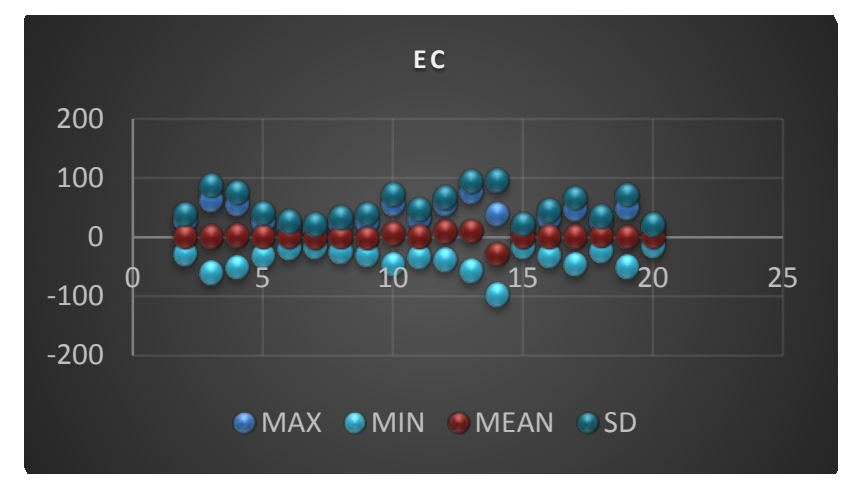

Figure 10. Participant 3

C. MAXIMUM AND MINIMUM CHANNEL FOR EYES CLOSE

Figure 7. Participant 3

\section{B. EYES OPEN}

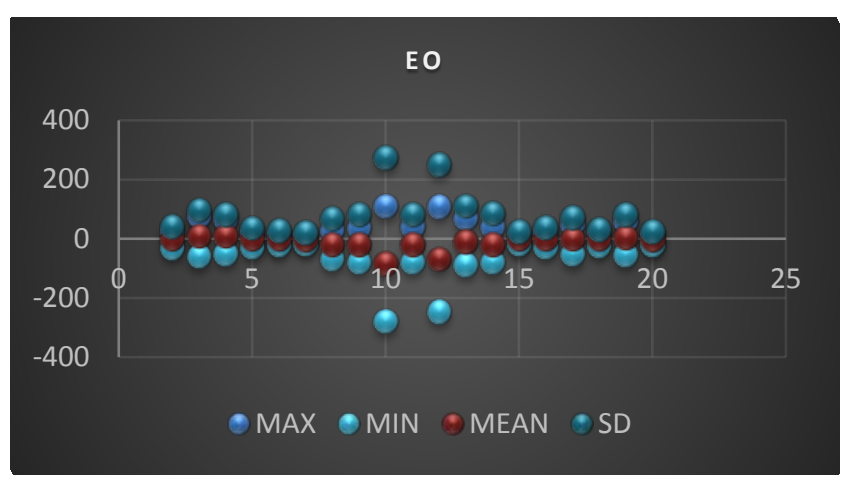

Figure 8. Participant 1
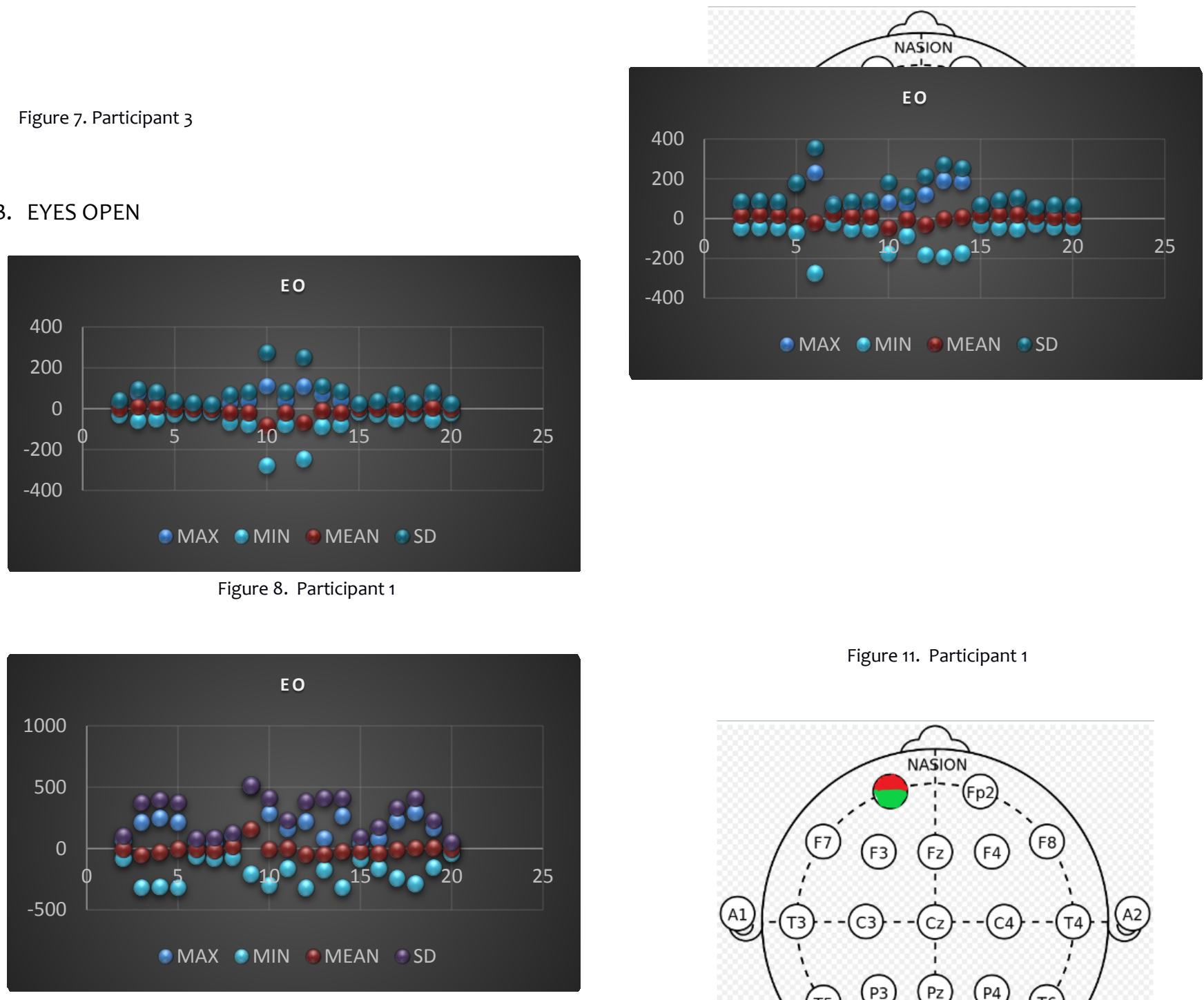

Figure 9. Participant 2

Figure 11. Participant 1

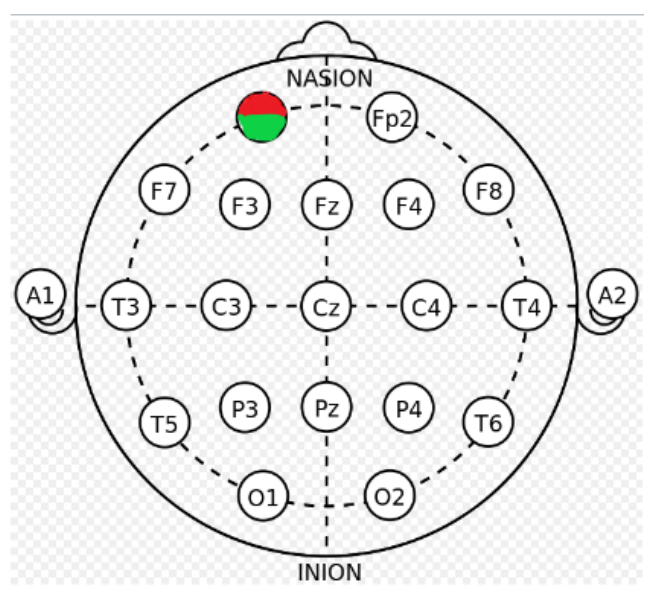

Figure 12. Participant 2 


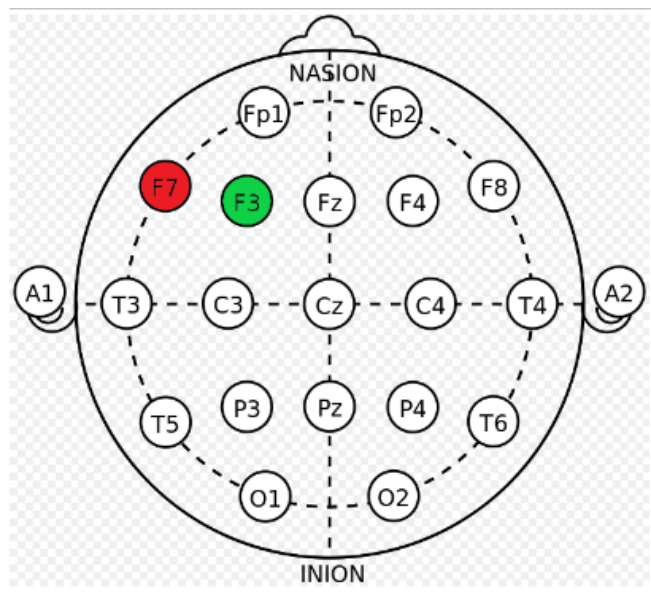

Figure 13. Participant 3

\section{MAXIMUM AND MINIMUM CHANNEL FOR EYES OPEN}

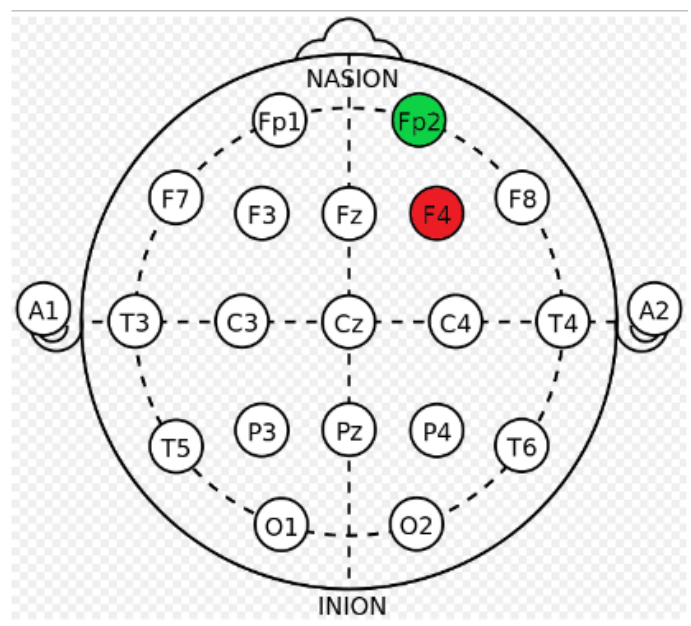

Figure 14. Participant 1

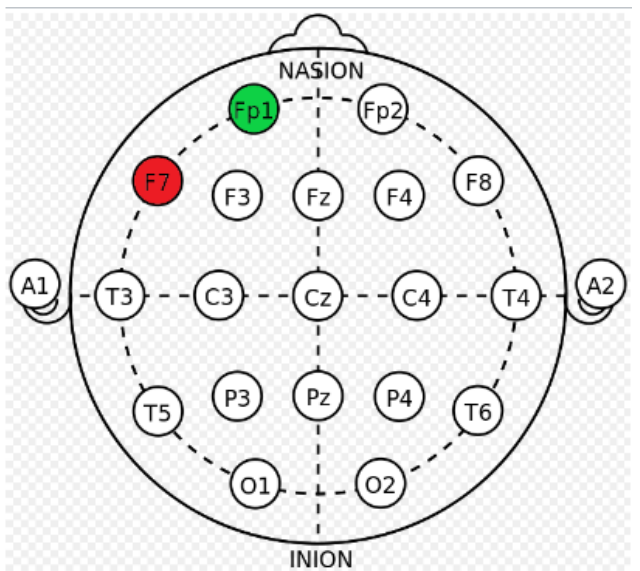

Figure 15. Participant 2

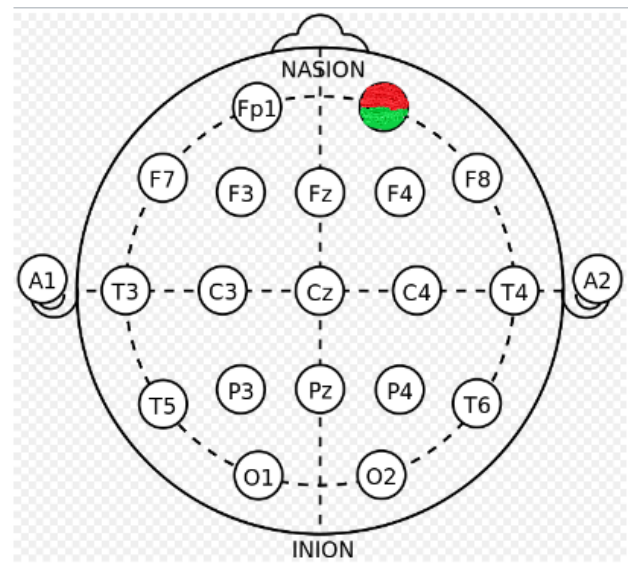

Figure 16. Participant 3

Figure 5,6 and 7 shows the plot based on features for maximum, minimum, mean and standard deviation for eyes close. $X$ axis refer to the channel number where the $Y$ axis is referred to the Value based on features. From the plot, we could observe only the pattern. It seems that identical pattern for participant 2 and participant 3 where the maximum range is not exceeded over 200.Compared to participant 1 which is almost reach to 500 in value. For the minimum value, all three participants share the same range of value which is below 200.The mean value for three participants show a linear value according to the plot. Nevertheless, for the standard deviation, the pattern seems scattered mostly for participant 2.

Whereas figure 8,9 and 10 shows the plot based on features for maximum, minimum, mean and standard deviation for eyes open. Also, it seems that identical pattern for participant 2 and participant 3 where the maximum range is not exceeded over 400.Compared to participant 1 which is exceeded over 500 in value. For the minimum value, all three participants share the same range of value which is 
below 400.The mean value for three participants also show a linear value according to the plot. Nevertheless, for the standard deviation, the pattern seems scattered mostly for participant 1.

Figure 11,12 and 13 shows the maximum and minimum channel positioning for eyes close. All three participants did not share the similarity for the minimum and maximum positioning channel. Participant 1 show the maximum at channel F4 and minimum at T5 or P7. But for participant 2 shared the same channel for maximum and minimum, located at Fp1.Then the maximum and minimum channel for participant 3 located at F7 for maximum and F3 for minimum.

Figure 14,15 and 16 shows the maximum and minimum channel positioning for eyes open. For eyes open also all three participants did not share the similarity for the minimum and maximum positioning channel. Participant 1 show the maximum at channel Fp2 and minimum at F4. Then for participant 2, the maximum at channel Fp1 and minimum at F7. But for participant 3 shared the same channel for maximum and minimum, located at FP2.

\section{CONCLUSSIONS}

From the observation, most of the maximum and minimum channel located at the frontal region when look at the channel positioning. For the shared value at the same channel for maximum and minimum also located at the frontal region of brain. The frontal area of the brain is where human affective state control, judgment, and insight comes from. This study expect that this type of pattern probably had a significant related to stress. Because the major areas of the brain most affected by stress is the prefrontal cortex, at the frontal region of brain [3]. This need to an extend of study to confirm the expectation.

To extend this study, future work could extend the emotion classification based on Valence and Arousal in detail to see which channel had the higher accuracy and then compare with the psychological stress measurement method (questionnaire) for example, probably with Dass 21 questionnaire.

\section{ACKNOWLEDGMENTS}

The authors would like to thank Dr. Manivannam from ALAM for arranging the session for the simulation test, run by two Captains pilot. Both were helping in the testing of this device. We would like to thank to PCBDG Research Center for allowing to use their EXG brain marker device. This work is supported by Fundamental Research Grant Scheme (FRGS) funded by the Ministry of Higher Education (Grant code: FRGS14-136-0377).

\section{REFERENCES}

[1] Aaronson, B. Electroencephalogram (EEG). In Encyclopedia of Autism Spectrum Disorders, Springer New York,2013, pp. 1067-1068.

[2] Adnan, Hafizuddin Muhd, et al. "Revolving Traditional EEG Device into Mobile Architecture." Information and Communication Technology for The Muslim World (ICT4M), 2016 6th International Conference on. IEEE, 2016.

[3] Alkadhi, Karim. "Brain physiology and pathophysiology in mental stress." ISRN Physiology 2013 (2013).

[4] American Psychological Association. "Stress in America: Our health at risk." Washington DC, American Psychological Association (2012).

[5] Baum, Andrew. "Stress, intrusive imagery, and chronic distress." Health psychology 9.6 (1990): 653.

[6] Belmonte, Matthew K., et al. "Autism and abnormal development of brain connectivity." Journal of Neuroscience24.42 (2004): 9228-9231.

[7] Brewin, Chris R. "Episodic memory, perceptual memory, and their interaction: Foundations for a theory of posttraumatic stress disorder." Psychological Bulletin 140.1 (2014): 69.

[8] Cohen, Sheldon, and Denise Janicki-Deverts. "Who's stressed? Distributions of psychological stress in the United States in probability samples from 1983, 2006, and 2009." Journal of applied social psychology 42.6 (2012): 1320-1334.

[9] Cousino, Melissa K., and Rebecca A. Hazen. "Parenting stress among caregivers of children with chronic illness: a systematic review." Journal of pediatric psychology 38.8 (2013): 809-828.

[10] Gargiulo, Gaetano, et al. "A mobile EEG system with dry electrodes." Biomedical Circuits and Systems Conference, 2008. BioCAS 2008. IEEE. IEEE, 2008.

[11] Giannakakis, Giorgos, Dimitris Grigoriadis, and Manolis Tsiknakis. "Detection of stress/anxiety state from EEG features during video watching." Engineering in Medicine and Biology Society (EMBC), 2015 37th Annual International Conference of the IEEE. IEEE, 2015.

[12] Guger, Christoph, et al. "Prosthetic control by an EEG-based braincomputer interface $(\mathrm{BCl})$." Proc. aaate 5 th european conference for the advancement of assistive technology. 1999.

[13] Hanson, Jamie L., et al. "Behavioral problems after early life stress: contributions of the hippocampus and amygdala." Biological psychiatry 77.4 (2015): 314-323.

[14] Hernandez, Javier, et al. "Under pressure: sensing stress of computer users." Proceedings of the SIGCHI conference on Human factors in computing systems. ACM, 2014.

[15] Hosseini, Seyyed Abed, and Mohammad Ali Khalilzadeh. "Emotional stress recognition system using EEG and psychophysiological signals: Using new labelling process of EEG signals in emotional stress state." Biomedical Engineering and Computer Science (ICBECS), 2010 International Conference on. IEEE, 2010.

[16] II'luchenok, I. R., A. N. Savost'lanov, and R. G. Valeev. "Dynamics of spectral characteristics of theta-and alpha-range EEG during negative emotional reactions." Zhurnal vysshei nervnoi deiatelnosti imeni IP Pavlova 51.5 (2001): 563-571.

[17] Jaušovec, Norbert, Ksenija Jaušovec, and Ivan Gerlič. "Differences in event-related and induced EEG patterns in the theta and alpha frequency bands related to human emotional intelligence." Neuroscience Letters 311.2 (2001): 93-96.

[18] Jenke, Robert, Angelika Peer, and Martin Buss. "Feature extraction and selection for emotion recognition from EEG." IEEE Transactions on Affective Computing 5.3 (2014): 327-339.

[19] Ko, Li-Wei, et al. "Mobile EEG \& ECG integration system for monitoring physiological states in peforming simulated war game training." Computational Intelligence and Games (CIG), 2015 IEEE Conference on. IEEE, 2015.

[20] Kranner, Ilse, et al. "What is stress? Concepts, definitions and applications in seed science." New Phytologist 188.3 (2010): 655-673.

[21] Kunze, Kai, et al. "Reading Activity Recognition Using an Off-the-Shelf EEG--Detecting Reading Activities and Distinguishing Genres of Documents." Document Analysis and Recognition (ICDAR), 2013 12th International Conference on. IEEE, 2013. 
[22] Lang, Peter J. "The emotion probe: Studies of motivation and attention." American psychologist 50.5 (1995): 372.

[23] Lang, Peter J. "International affective picture system (IAPS): Affective ratings of pictures and instruction manual." Technical report (2005).

[24] Liao, Lun-De, et al. "Human cognitive application by using wearable mobile brain computer interface." TENCON 2010-2010 IEEE Region 10 Conference. IEEE, 2010.

[25] Lin, Chin-Teng, et al. "Real-time assessment of vigilance level using an innovative Mindo 4 wireless EEG system." Circuits and Systems (ISCAS), 2013 IEEE International Symposium on. IEEE, 2013.

[26] Lin, Yuan-Pin, Yijun Wang, and Tzyy-Ping Jung. "A mobile SSVEP-based brain-computer interface for freely moving humans: The robustness of canonical correlation analysis to motion artifacts." Engineering in Medicine and Biology Society (EMBC), 2013 35th Annual International Conference of the IEEE. IEEE, 2013.

[27] Marsella, Stacy, and Jonathan Gratch. "Computationally modeling human emotion." Communications of the ACM 57.12 (2014): 56-67.

[28] Mehrabian, Albert, and James A. Russell. An approach to environmental psychology. the MIT Press, 1974.

[29] Niedermeyer, Ernst, and FH Lopes da Silva, eds. Electroencephalography: basic principles, clinical applications, and related fields. Lippincott Williams \& Wilkins, 2005.

[30] Othmana, Marini, et al. "EEG Emotion Recognition Based On The Dimensional Models Of Emotions." The 9th International Conference on Cognitive Science, in Procedia-Social and Behavioral Sciences. Vol. 97. 2013.

[31] Otto, Chris, et al. "System architecture of a wireless body area sensor network for ubiquitous health monitoring." Journal of mobile multimedia 1.4 (2006): 307-326.

[32] Saeed, Sanay Muhammad Umar, et al. "Psychological stress measurement using low cost single channel EEG headset." Signal Processing and Information Technology (ISSPIT), 2015 IEEE International Symposium on. IEEE, 2015.

[33] Shams, Wafaa Khazaal, Abdul Wahab, and Imad Fakhri. "Affective computing model using source-temporal domain." Procedia-Social and Behavioral Sciences 97 (2013): 54-62.

[34] Sharma, Nandita, and Tom Gedeon. "Modeling stress recognition in typical virtual environments." Pervasive Computing Technologies for Healthcare (PervasiveHealth), 2013 7th International Conference on. IEEE, 2013.

[35] Sinclair, Samuel Justin, et al. "Psychometric evaluation and normative data for the depression, anxiety, and stress scales-21 (DASS-21) in a nonclinical sample of US adults." Evaluation \& the Health Professions 35.3 (2012): 259-279.

[36] Smith, Craig A., and Phoebe C. Ellsworth. "Patterns of appraisal and emotion related to taking an exam." Journal of personality and social psychology 52.3 (1987): 475.

[37] Stam, C. J., et al. "Graph theoretical analysis of magnetoencephalographic functional connectivity in Alzheimer's disease." Brain 132.1 (2008): 213-224.

[38] Sulaiman, Norizam, et al. "Intelligent system for assessing human stress using EEG signals and psychoanalysis tests." Computational Intelligence, Communication Systems and Networks (CICSyN), 2011 Third International Conference on. IEEE, 2011.

[39] Sullivan, Thomas J., et al. "A brain-machine interface using drycontact, low-noise EEG sensors." Circuits and Systems, 2008. ISCAS 2008. IEEE International Symposium on. IEEE, 2008.

[40] Tamagawa, Rie, et al. "Trait mindfulness, repression, suppression, and self-reported mood and stress symptoms among women with breast cancer." Journal of clinical psychology 69.3 (2013): 264-277.

[41] Vignola, Rose Claudia Batistelli, and Adriana Marcassa Tucci. "Adaptation and validation of the depression, anxiety and stress scale (DASS) to Brazilian Portuguese." Journal of affective disorders 155 (2014): 104-109.

[42] Yik, Michelle SM, James A. Russell, and Lisa Feldman Barrett. "Structure of self-reported current affect: Integration and beyond." Journal of personality and social psychology77.3 (1999): 600. 